\title{
Effects of mental health and substance use disorder symptoms on the association between STDs and sexual identity: evidence from the 2017-2019 National Survey on Drug Use and Health
}

\author{
David Adzrago $^{1}$ (D - Samuel H. Nyarko ${ }^{2}$ Nnenna Ananaba ${ }^{1} \cdot$ Christine Markham $^{1}$
}

Received: 17 June 2021 / Accepted: 19 November 2021

This is a U.S. government work and not under copyright protection in the U.S.; foreign copyright protection may apply 2021

\begin{abstract}
Background Sexually transmitted disease (STD) cases are rising in the USA, especially among sexual and gender minorities, despite the availability of numerous STD prevention programs. We examined the differences in STD prevalence among sexual and gender minority subgroups with major depressive episode symptoms and substance use dependence.

Methods We combined 2017, 2018, and 2019 National Survey on Drug Use and Health (NSDUH) public-use data on adults $(N=127,584)$ to conduct weighted multivariable logistic regression and margins analyses.

Results Approximately $2.05 \%$ of the population reported having STDs. The population that had major depressive episode symptoms $(\mathrm{AOR}=1.70,95 \% \mathrm{CI}=1.46,1.99)$, alcohol use dependence $(\mathrm{AOR}=1.79,95 \% \mathrm{CI}=1.49,2.16)$, illicit drug use other than marijuana use dependence $(\mathrm{AOR}=2.25,95 \% \mathrm{CI}=1.73,2.92)$, or marijuana use dependence $(\mathrm{AOR}=1.90$, $95 \% \mathrm{CI}=1.57,2.31)$ had higher odds of contracting STDs compared to their counterparts. Lesbian/gay (AOR $=2.81,95 \%$ $\mathrm{CI}=2.24,3.54)$ and bisexual ( $\mathrm{AOR}=1.95,95 \% \mathrm{CI}=1.60,2.37)$ individuals had higher odds of contracting STDs. Lesbians/ gays with major depressive episode symptoms, alcohol use dependence, or illicit drug use other than marijuana use dependence had the highest probability of having STDs, compared to bisexuals and heterosexuals with major depressive episode symptoms, alcohol use, or illicit drug use other than marijuana use dependence. Bisexuals with marijuana use dependence had the highest probability of STD contraction compared to their lesbian/gay and heterosexual counterparts. Within each sexual identity subgroup, the probability of having STDs was higher for individuals with major depressive episode symptoms, or dependence on alcohol use, illicit drug use other than marijuana use, or marijuana use compared to their counterparts. Conclusion Major depressive episode symptoms, substance use dependence, and sexual and gender minority status had higher risks for STD diagnosis, particularly for sexual and gender minorities with major depressive episode symptoms or substance use dependence. Tailored interventions based on major depressive episode symptoms and substance use dependence may reduce the prevalence of STD, especially among sexual and gender minorities.
\end{abstract}

Keywords STDs $\cdot$ Sexual minority $\cdot$ Depression $\cdot$ Substance use disorders

\section{Introduction}

There is a growing rise in sexually transmitted disease (STD) cases reported in the US (CDC 2021; Shannon and Klausner

David Adzrago

David.Adzrago@uth.tmc.edu

1 Center for Health Promotion and Prevention Research (CHPPR), School of Public Health, The University of Texas Health Science Center at Houston (UTHealth), 7000 Fannin, Suite 2502A, Houston, TX 77030, USA

2 Department of Epidemiology, Human Genetics \& Environmental Sciences, UTHealth School of Public Health, Houston, TX, USA
2018) and worldwide (World Health Organization 2021).

The World Health Organization reports more than a million STD infections each day worldwide (World Health Organization 2021), while US data shows approximately 20 million new STD infections each year (Healthy People 2021; Shannon and Klausner 2018). In 2018, the CDC reported 26 million new STDs totaling nearly 68 million infections in the USA (CDC 2021). This implies that 1 in 5 people in the USA had an STD on any given day in 2018 (CDC 
2021). When not detected or treated early, STDs cause harmful, sometimes, irreversible and costly clinical implications (Healthy People 2021; Magidson et al. 2014). STDs such as syphilis, chlamydia, gonorrhea, and HPV are known to cause significant health problems, including reproductive health problems, chronic pelvic pain, cervical cancer, ectopic pregnancy, and facilitation of HIV infection (Healthy People 2021; Magidson et al. 2014). In 2018, STDs accounted for an estimated $\$ 16$ billion in direct medical costs, while chlamydia, gonorrhea, and syphilis alone contributed $\$ 1.1$ billion in total medical costs (CDC 2021). Women contributed $25 \%$ of the total costs, while youths aged 15-24 contributed $26 \%$ of the total costs, not accounting for the costs associated with other non-medical costs, STD prevention, and lost productivity (CDC 2021).

Although studies have examined the association between STDs and their potential risk factors, these studies used only treatment-seeking and homeless samples (Carey et al. 2004; Rein et al. 2004; Erbelding et al. 2001; Rohde et al. 2001), sample sizes with limited statistical power (Erbelding et al. 2001), and limited age ranges such as only adolescents (Rohde et al. 2001; Ramrakha et al. 2000). Also, most of these studies are relatively old and therefore may not reflect the current dynamics of STD risks. The spread of STDs may be associated with factors such as sexual and gender identity, gender, age, race/ethnicity, income, mental health disorders, and substance use (Healthy People 2021; Kaiser Family Foundation 2021; Shannon and Klausner 2018; Feaster et al. 2016; Rein et al. 2004). Such factors may accelerate the risk of STD infections and trigger severe impediments to STD prevention due to their influence on social and sexual networks, social norms regarding sex and sexuality as well as willingness to seek care (Healthy People 2021; Kaiser Family Foundation 2021; Feaster et al. 2016; Rein et al. 2004). A study found that women who have sex with women (WSW) were more likely to have a mental health disorder compared to women who have sex with men (WSM) (Reisner et al. 2010). However, this study included only female participants and was conducted in Boston, MA, which may not reflect the association in the general U.S. population. Individuals with mental health disorders such as depression have been shown to be at high risk for STD infections (Rein et al. 2004; Erbelding et al. 2001; Otto-Salaj and Stevenson 2001). However, these studies included only privately insured people aged 15-44 years, only HIV-related risks, and convenience samples. Compared to White Americans, Black or African Americans and people living in poverty may be at greater risks of having STDs, which may be due to limited access to and use of healthcare (CDC 2019; Hogben and Leichliter 2008; Newman and Berman 2008). Furthermore, individuals reporting substance use such as alcohol and illicit drug use have been found to engage in risky sexual behaviors, which may increase their risk of STD infections (Kaiser Family
Foundation 2021; Harbertson et al. 2019; Feaster et al. 2016; Dembo et al. 2009). While previous studies have identified an association between substance use and STD contraction, there is variation in association due to drug type, sexual networks, and other factors (Feaster et al. 2016). For instance, Carey et al. (2004) found in a psychiatric outpatient sample that heavy alcohol use was associated with multiple partners for men who have sex with men (MSM) as well as women but not for heterosexual men. This suggests increased sexual risk behaviors for MSM and women concerning alcohol use but not heterosexual men. Studies have also reported an association with cocaine use but not heroin use and higher risk behavior scores (Feaster et al. 2016). Other studies have reported gender disparity on the association between marijuana use and STD contraction where females are more disproportionately affected compared to males (Feaster et al. 2016; Dembo et al. 2009). These inconsistencies may be due to samples with limited age and description (e.g., juvenile with an arrest history, military personnel, patients in treatments) (Harbertson et al. 2019; Dembo et al. 2009).

To bridge the gap in the literature, we used a nationally representative sample of adults aged 18 years or older to: (1) assess the prevalence of STD by mental health disorder symptoms, substance use dependence, and sociodemographic characteristics; (2) examine the association between STD diagnosis status and mental health disorder symptoms, substance use dependence, sexual and gender identity, and sociodemographic characteristics; and (3) evaluate differences in STD diagnosis status between and within sexual identity groups by mental health disorder symptoms and substance use dependence. The results from this study could inform the development of tailored public health interventions based on mental health disorder symptoms and substance use dependence to treat, reduce, and prevent STDs in the population, especially among sexual and gender minority individuals.

\section{Methods}

\section{Study design}

We combined 2017, 2018, and 2019 National Survey on Drug Use and Health (NSDUH) de-identified public-use data for this study. Details of the construction of the samples and survey questions and administration can be found in the Center for Behavioral Health Statistics and Quality (2020a, b) and Substance Abuse and Mental Health Services Administration ([SAMHSA] 2020). The NSDUH is an annual cross-sectional survey that assesses substance use and mental health issues among the U.S. population aged 12 years and above. Each respondent receives an incentive of \$30. NSDUH uses a complex, multistage area probability 
sample of the U.S. civilian, noninstitutionalized population in each of the 50 states and the District of Columbia. NSDUH requires that the data be weighted to obtain unbiased population estimates because the NSDUH includes a representative sample of the population rather than the entire population. The combined data of the 2017, 2018, and 2019 surveys consist of a total of 168,725 individuals aged 12 years or older. We, however, conducted our analysis on adults aged 18 years or older, using a total of 127,584 adults with complete data on sexually transmitted disease (STD) diagnosis status. We pooled data across years to increase the sample size and statistical power.

\section{Measures}

\section{Dependent variable}

The dependent variable is sexually transmitted disease (STD) status, which was self-reported. The participants were asked to indicate whether they had an STD (i.e., chlamydia, gonorrhea, herpes, or syphilis) in the past 12 months (yes/ no).

\section{Independent variables}

We examined four main independent variables: (1) major depressive episode (MDE) symptoms in the past year, and (2) past-year alcohol use dependence, (3) past-year illicit drugs other than marijuana dependence, and (4) past-year marijuana use dependence. MDE symptoms were assessed in the NSDUH based on the Diagnostic and Statistical Manual of Mental Disorders 5th edition ([DSM-5] American Psychiatric Association 2013; Harvard School of Medicine 2005). MDE questions measured if participants had experienced an MDE in the past year (see details in Center for Behavioral Health Statistics and Quality 2020a, b). Based on self-report by the participant on the DSM-5 questions, the participant was classified by the NSDUH as having MDE in the past year if that participant had a lifetime MDE and a time in the past 12 months when that participant felt depressed or lost interest or pleasure in daily activities for 2 weeks or longer, while also having some of the other symptoms for lifetime MDE. Else, the participant was classified by the NSDUH as not having MDE in the past year if that participant had no lifetime MDE or with lifetime MDE but no period of depression lasting 2 weeks or longer while having other symptoms for lifetime MDE.

Alcohol use dependence was assessed with questions based on DSM-4 criteria (SAMHSA 2020; American Psychiatric Association 1994). A participant was classified with alcohol use dependence if the participant met three or more of the seven DSM-4 alcohol use dependence criteria. Otherwise, the participant was classified as not having alcohol use dependence. These criteria include: (1) Spent a great deal of time over a month or more getting, using, or getting over the effects of alcohol, (2) Used alcohol more often than intended or was unable to keep set limits on alcohol use, (3) Needed to use alcohol more than before to get desired effects or noticed that same amount of alcohol use had less effect than before, (4) Inability to cut down or stop using alcohol every time tried or wanted to, (5) Continued to use alcohol even though it was causing problems with emotions, nerves, mental health, or physical problems, (6) Alcohol use reduced or eliminated involvement or participation in important activities, (7) Reported experiencing two or more alcohol withdrawal symptoms at the same time that lasted longer than a day after alcohol use was cut back or stopped.

Illicit drug use dependence other than marijuana use was ascertained based on dependence on other illicit substances (SAMHSA 2020). A participant was classified with illicit drug use dependence other than marijuana use if the participant was categorized as dependent on at least one of the following substances: hallucinogens, inhalants, methamphetamine, cocaine, heroin, prescription pain relievers, prescription sedatives, prescription stimulants, or prescription tranquilizers. Otherwise, the participant was classified with no illicit drug use dependence other than marijuana use.

Marijuana use dependence was assessed based on six DSM-4 marijuana use dependence criteria (SAMHSA 2020; American Psychiatric Association 1994). A participant was classified with marijuana use dependence if the participant met three or more of the six criteria. Else, the participant was classified as not having marijuana use dependence. The criteria are: (1) Spent a great deal of time over a month or more getting, using, or getting over the effects of marijuana, (2) Used marijuana more often than intended or was unable to keep set limits on marijuana use, (3) Needed to use marijuana more than before to get desired effects or noticed that same amount of marijuana use had less effect than before, (4) Inability to cut down or stop using marijuana every time tried or wanted to, (5) Continued to use marijuana even though it was causing problems with emotions, nerves, mental health, or physical problems, and (6) Marijuana use reduced or eliminated involvement or participation in important activities.

\section{Covariates}

The following self-reported sociodemographic characteristics were included in our study based on their potential association with STDs in previous studies (Coyle et al. 2019; Santos et al. 2018; Tao 2008): age (18-25, 26-34, 35-49, 50-64, 65 $\geq$ ), sex (male/female), sexual identity (heterosexual, lesbian or gay, and bisexual), race/ethnicity (nonHispanic White, non-Hispanic Black/African American, Hispanic, and Other race [non-Hispanic Native American/ 
Alaskan Native, non-Hispanic Asian American, non-Hispanic Native Hawaiian/Other Pacific Islander, and non-Hispanic more than one race]), level of education completed (12th grade or less grade, High School diploma/GED, some college credit but no degree, Associate's degree, and college graduate or higher), total family income $(<\$ 20,000 ; \$ 20,000$ to $\$ 49,999 ; \$ 50,000-\$ 74,999$; and $\geq \$ 75,000)$, employment status (employed full time, part time, unemployed, or other [including those not in labor force]), and county metro/nonmetro (large metro, small metro, and non-metro). These variables were analyzed as categorical variables in our study.

\section{Statistical analyses}

STATA/SE, version 16.1 (StataCorp 2019), was applied to perform data analysis. Descriptive statistics and bivariate analysis were performed to describe unweighted frequencies and weighted percentages of the participants' sociodemographic characteristics, and the prevalence of STD diagnosis status, MDE symptoms, alcohol use dependence, illicit drug use dependence other than marijuana use, and marijuana use dependence (see Table 1). The bivariate analyses assessed the association between STD diagnosis status and sociodemographic characteristics, MDE symptoms, alcohol use dependence, illicit drug use dependence other than marijuana use, and marijuana use dependence, respectively, using chi-square tests at a significance level of 0.05 . All the statistically significant variables at the bivariate analysis level $(p<0.05)$ were entered into the multivariable logistic regression model (see Table 2). We adjusted for the sociodemographic variables in the multivariable logistic regression model examining the association between STD diagnosis status and MDE symptoms, alcohol use dependence, illicit drug use dependence other than marijuana use, and marijuana use dependence. We also examined the interaction between sexual identity and MDE symptoms, alcohol use dependence, illicit drug use dependence other than marijuana use, and marijuana use dependence, respectively, using joint significant effects with the Adjusted Wald test. We found significant interaction results (all $p s<0.001$ ). Based on the interaction effects, we further performed margins analyses to examine between and within-groups differences in STD diagnosis status (shown in Figs. 1-4). All the analyses were weighted using the NSDUH survey weight to obtain nationally representative estimates. The NSDUH survey weight helped to obtain weighting and clustering effects such as unequal probabilities of sampling, non-response, and post-stratification adjustments (Center for Behavioral Health Statistics and Quality 2020a, b). The NSDUH nesting variables were also used to capture explicit stratification and to ascertain clustering with the data and obtain accurate variance estimates (Center for Behavioral Health Statistics and Quality 2020a, b). NSDUH includes imputed variables such as income variables and recommends their use (Center for Behavioral Health Statistics and Quality 2020b). We therefore used all the imputed variables (including income) as applicable to our study. We reported adjusted odds ratios (AORs) with 95\% confidence intervals (95\% CIs), percentages with $95 \%$ CIs, and considered statistically significant results at $\mathrm{p}<0.05$.

\section{Results}

\section{Descriptive and bivariate statistics}

Descriptive and bivariate statistics of the study sample (unweighted $N=127,584$ and weighted $N=247,549,178$ ) are presented in Table 1. Most of the population was within ages 50-64 (25.09\%), females (51.77\%), non-Hispanic Whites (63.53\%), heterosexuals $(94.55 \%)$, had college graduate or higher degrees $(32.43 \%), \$ 75,000$ or more (39.64\%), employed full time (49.73\%), and resided in a large metropolitan area (55.88\%). Significant proportions of the population had STDs (2.05\%), alcohol use dependence $(3.12 \%)$, illicit drug use other than marijuana use dependence (1.29\%), marijuana use dependence (1.08\%), and MDE symptoms (7.42\%). Of those who had STDs, the majority were within ages 35-49 (23.89\%), females $(59.85 \%)$, nonHispanic Whites (62.89\%), heterosexual (84.24\%), had college graduate or higher degree $(30.81 \%), \$ 75,000$ or more $(34.60 \%)$, and were employed full time (52.71\%). Significant proportions of those who had STDs had MDE symptoms (17.36\%) were dependent on alcohol use (8.34\%), illicit drug use other than marijuana use (5.05\%), and marijuana use $(4.11 \%)$. The bivariate statistics show that age, sex, race/ ethnicity, sexual identity, education, total family income, employment status, MDE symptoms, alcohol use dependence, illicit drug use other than marijuana use dependence, and marijuana use dependence were associated with STDs diagnosis status. These significant variables at the bivariate analysis level were entered in the weighted multivariable logistic regression model.

\section{Multivariable logistic regression analysis}

Table 2 presents the weighted multivariable logistic regression analysis results. The results showed that having had MDE symptoms $(\mathrm{AOR}=1.70,95 \% \mathrm{CI}=1.46,1.99)$, alcohol use dependence $(\mathrm{AOR}=1.79,95 \% \mathrm{CI}=1.49,2.16)$, illicit drug use other than marijuana use dependence $(\mathrm{AOR}=2.25$, 95\% CI $=1.73,2.92$ ), or marijuana use dependence $(\mathrm{AOR}=1.90,95 \% \mathrm{CI}=1.57,2.31)$ was associated with higher odds of having STDs compared to those without MDE symptoms or substance use dependence. Compared to individuals aged $18-25$ years, those within ages $35-49$ 
Table 1 Self-reported STD diagnosis status by participants' sociodemographic characteristics, substance use dependence status, and MDE: a weighted sample of U.S. adults (unweighted $N=127,584$ ), NSDUSH 2017-2019

\begin{tabular}{|c|c|c|c|c|}
\hline & $\begin{array}{l}\text { Overall } \\
\mathrm{N}(\%[95 \% \mathrm{CI}])\end{array}$ & $\begin{array}{l}\text { No STDs } \\
\mathrm{n}(\%[95 \% \mathrm{CI}])\end{array}$ & $\begin{array}{l}\text { STDs } \\
\mathrm{n}(\%[95 \% \mathrm{CI}])\end{array}$ & $p$ value \\
\hline & & $124,294(97.95[97.85-98.05])$ & $3290(2.05[1.95-2.15])$ & \\
\hline \multicolumn{5}{|l|}{ Covariates } \\
\hline Age & & & & $<.001$ \\
\hline $18-25$ & $41,366(13.64[13.43-13.85])$ & $39,887(13.45[13.24-13.66])$ & $1479(22.81[21.17-24.54])$ & \\
\hline $26-34$ & $26,035(16.05[15.72-16.38])$ & $25,302(15.93[15.60-16.27])$ & $733(21.82[19.55-24.27])$ & \\
\hline $35-49$ & $33,874(24.53[24.17-24.90])$ & $33,177(24.54[24.18-24.91])$ & $697(23.89[21.93-25.97])$ & \\
\hline $50-64$ & $14,761(25.09[24.66-25.52])$ & $14,510(25.17[24.75-25.59])$ & $251(21.29[18.47-24.42])$ & \\
\hline 65 or older & $11,548(20.70[20.2-21.201])$ & $11,418(20.92[20.41-21.43])$ & $130(10.19[8.41-12.29])$ & \\
\hline Sex & & & & $<.001$ \\
\hline Male & $59,696(48.23[47.82-48.64])$ & $58,529(48.40[47.99-48.81])$ & $1167(40.15[37.48-42.89])$ & \\
\hline Female & $67,888(51.77[51.36-52.18])$ & $65,765(51.60[51.19-52.01])$ & $2123(59.85[57.11-62.52])$ & \\
\hline Race/ethnicity & & & & $<.001$ \\
\hline Non-Hispanic White & $76,671(63.53[62.87-64.19])$ & $74,810(63.55[62.88-64.21])$ & $1861(62.89[60.33-65.37])$ & \\
\hline $\begin{array}{l}\text { Non-Hispanic Black/African Ameri- } \\
\text { can }\end{array}$ & $15,924(11.85[11.37-12.35])$ & $15,315(11.76[11.27-12.26])$ & $609(16.35[14.36-18.56])$ & \\
\hline Hispanic & $22,118(16.24[15.77-16.72])$ & $21,629(16.29[15.82-16.78])$ & $489(13.67[12.06-15.46])$ & \\
\hline Other race & $12,871(8.38[8.11-8.65])$ & $12,540(8.40[8.14-8.68])$ & $331(7.09[6.06-8.29])$ & \\
\hline Sexual identity & & & & $<.001$ \\
\hline Heterosexual (i.e., straight) & $115,696(94.55[94.36-94.73])$ & $113,026(94.77[94.58-94.95])$ & $2670(84.24[82.37-85.95])$ & \\
\hline Lesbian or Gay & $2849(1.98[1.86-, 2.10])$ & $2688(1.89[1.78-2.01])$ & $161(5.94[4.83-7.28])$ & \\
\hline Bisexual & $6814(3.48[3.35-3.60])$ & $6388(3.34[3.22-3.47])$ & $426(9.82[8.44-11.41])$ & \\
\hline Level of education completed & & & & .002 \\
\hline Twelfth grade or less grade & $15,887(12.12[11.79-12.46])$ & $15,510(12.18[11.83-12.53])$ & $377(9.59[8.32-11.03])$ & \\
\hline High School diploma/GED & $33,704(24.48[24.05-24.92])$ & $32,791(24.49[24.05-24.94])$ & $913(24.14[22.59-25.76])$ & \\
\hline Some college credit but no degree & $30,959(21.65[21.35-21.95])$ & $30,032(21.56[21.26-21.87])$ & $927(25.80[23.26-28.52])$ & \\
\hline Associate's degree & $11,892(9.32[9.06-9.58])$ & $11,604(9.31[9.05-9.58])$ & $288(9.67[8.00-11.64])$ & \\
\hline College graduate or higher & $35,142(32.43[31.78-33.08])$ & $34,357(32.46[31.80-33.13])$ & $785(30.81[28.29-33.45])$ & \\
\hline Total family income & & & & $<.001$ \\
\hline Less than $\$ 20,000$ & $24,055(15.48[15.12-15.86])$ & $23,229(15.40[15.03-15.77])$ & $826(19.62[17.85-21.52])$ & \\
\hline$\$ 20,000-\$ 49,999$ & $39,181(29.07[28.55-29.61])$ & $38,092(29.04[28.53-29.56])$ & 1089 (30.58[27.91-33.39]) & \\
\hline$\$ 50,000-\$ 74,999$ & $20,110(15.80[15.48-16.13])$ & $19,652(15.81[15.49-16.15])$ & $458(15.20[13.36-17.24])$ & \\
\hline$\$ 75,000$ or more & $44,238(39.64[38.98-40.31])$ & $43,321(39.75[39.08-40.42])$ & $917(34.60[32.12-37.17])$ & \\
\hline Employment status & & & & $<.001$ \\
\hline Employed full time & $67,038(49.73[49.26-50.21])$ & $65,340(49.67[49.18-50.16])$ & $1698(52.71[49.20-56.21])$ & \\
\hline Employed part time & $19,811(12.98[12.69-13.29])$ & $19,213(12.92[12.62-13.22])$ & $598(16.09[14.33-18.02])$ & \\
\hline Unemployed & $7350(4.11[3.95-4.27])$ & $7047(4.05[3.89-4.21])$ & $303(7.02[5.93-8.30])$ & \\
\hline Other & $33,385(33.18[32.71-33.64])$ & $32,694(33.37[32.90-33.84])$ & $691(24.17[21.32-27.27])$ & \\
\hline County metro/non-metro area & & & & .057 \\
\hline Large metro & $57,223(55.88[55.20-56.551])$ & $55,705(55.83[55.15-56.50])$ & $1518(58.19[55.48-60.86])$ & \\
\hline Small metro & $45,146(29.88[29.26-30.50])$ & $43,942(29.88[29.27-30.50])$ & 1204 (29.58[27.01-32.29]) & \\
\hline Non-metro & $25,215(14.25[13.73-14.78])$ & $24,647(14.29[13.77-14.82])$ & 568 (12.22[10.69-13.95]) & \\
\hline \multicolumn{5}{|l|}{ Independent variables } \\
\hline Past year MDE symptoms & & & & $<.001$ \\
\hline No & $122,553(92.59[92.37-92.80])$ & $119,600(92.79[92.58-93.00])$ & $4,148,285(82.64[80.59-84.52])$ & \\
\hline Yes & $18,199,319(7.42[7.20-7.63])$ & $17,328,167(7.21[7.00-7.42])$ & $871,153(17.36[15.48-19.41])$ & \\
\hline Past year alcohol use dependence & & & & $<.001$ \\
\hline No & $122,553(96.89[96.72-97.04])$ & $119,600(96.99[96.83-97.16])$ & $2953(91.67[90.35-92.82])$ & \\
\hline Yes & $5031(3.12[2.96-3.28])$ & $4694(3.01[2.84-3.18])$ & $337(8.34[7.18-9.65])$ & \\
\hline
\end{tabular}


Table 1 (continued)

\begin{tabular}{llll}
\hline & $\begin{array}{l}\text { Overall } \\
\mathrm{N}(\%[95 \% \mathrm{CI}])\end{array}$ & $\begin{array}{l}\text { No STDs } \\
\mathrm{n}(\%[95 \% \mathrm{CI}])\end{array}$ & $\begin{array}{l}\text { STDs } \\
\mathrm{n}(\%[95 \% \mathrm{CI}])\end{array}$ \\
\hline $\begin{array}{l}\text { Past year illicit drug use other than } \\
\text { marijuana use }\end{array}$ & & & \\
No & $125,476(98.71[98.61-98.81])$ & $122,387(98.79[98.69-98.88])$ & $3089(94.95[93.80-95.89])$ \\
Yes & $2108(1.29[1.20-1.39])$ & $1907(1.21[1.12-1.31])$ & $201(5.05[4.11-6.20])$ \\
$\begin{array}{l}\text { Past year marijuana use dependence } \\
\text { No }\end{array}$ & $125,215(98.92[98.86-98.98])$ & $122,141(98.99[98.93-99.05])$ & $3074(95.89[95.08-96.57])$ \\
Yes & $2369(1.08[1.02-1.14])$ & $2153(1.01[0.95-1.07])$ & $216(4.11[3.44-4.92])$ \\
\hline
\end{tabular}

Data Source: National Survey on Drug Use and Health (NSDUH) 2019, 2018, and 2017

${ }^{1}$ Weighted $N=247,549,178$

${ }^{2}$ Statistical significance at $p<0.05$.

${ }^{3}$ All $p$-values are based on chi-square tests for the categorical variables.

${ }^{4} M D E$, Major depressive episode

${ }^{5} S T D$, Sexually transmitted diseases

$(\mathrm{AOR}=0.73,95 \% \mathrm{CI}=0.63,0.84), 50-64(\mathrm{AOR}=0.66$, $95 \% \mathrm{CI}=0.55,0.79)$, and 65 or older $(\mathrm{AOR}=0.44,95 \%$ $\mathrm{CI}=0.34,0.56)$ had lower odds of having STDs. Males had lower odds of having STDs (AOR $=0.73,95 \% \mathrm{CI}=0.65$, $0.82)$ compared to females. Compared to non-Hispanic Whites, non-Hispanic Black/African Americans had higher odds $(\mathrm{AOR}=1.29,95 \% \mathrm{CI}=1.09,1.54)$ while Hispanics $(\mathrm{AOR}=0.79,95 \% \mathrm{CI}=0.68,0.93)$ and other racial groups $(\mathrm{AOR}=0.82,95 \% \mathrm{CI}=0.68,0.98)$ had lower odds of having STDs. Those who self-identified as lesbian/gay $(\mathrm{AOR}=2.81,95 \% \mathrm{CI}=2.24,3.54)$ or bisexual $(\mathrm{AOR}=1.95$, $95 \% \mathrm{CI}=1.60,2.37$ ) individuals had higher odds of having STDs compared to heterosexual individuals. Individuals who had completed 12th grade or less, compared to college graduate or higher degree, had lower odds of having STDs $(\mathrm{AOR}=0.82,95 \% \mathrm{CI}=0.67,0.99)$, and high school diploma/GED $(\mathrm{OR}=1.30,95 \% \mathrm{CI}=1.07,1.57)$ versus college graduate or higher had higher odds of having STDs. Relative to those who had a total family income of less than $\$ 20,000$, those who had $\$ 75,000$ or more (AOR $=0.77,95 \%$ $\mathrm{CI}=0.67,0.89)$ had lower odds of having STDs. Individuals who reported their employment status as other (i.e., students, persons keeping house or caring for children full time, retired or disabled persons, or other persons not in the labor force) had lower odds of having STDs (AOR $=0.79,95 \%$ $\mathrm{CI}=0.66,0.95$ ) compared to those who were employed full time.

\section{Between and within-group analysis}

Figure 1 presents the differences in STDs diagnosis status between and within MDE symptoms and sexual identity groups. Individuals who self-identified as lesbians/gays and had MDE symptoms (8.67\%) had the highest probability of having STDs compared to bisexual (4.52\%) and heterosexual (3.13\%) individuals who also had MDE symptoms. The probability of having STDs was higher for individuals with MDE symptoms (3.13-8.67\%) than individuals without MDE symptoms (1.76-4.62\%) across all the sexual identity groups. Lesbian/gay individuals who had MDE symptoms had a higher probability of having STDs (8.67\%) than lesbian/gay individuals without MDE symptoms (4.62\%). Among bisexual individuals, those who had MDE symptoms had a higher probability of having STDs $(4.52 \%)$ than those without MDE symptoms (3.84\%). For heterosexual individuals, those who had MDE symptoms had a higher probability of having STDs (3.13\%) than those without MDE symptoms $(1.76 \%)$.

As shown in Fig. 2, lesbian/gay individuals who had alcohol use dependence had the highest probability of having STDs $(8.73 \%)$ compared to bisexual $(5.35 \%)$ and heterosexual (3.30\%) individuals who also had alcohol use dependence. Across the sexual identity groups, individuals who had alcohol use dependence had the highest probability of having STDs (3.30-8.73\%\%) compared to those without alcohol use dependence (1.83-4.91\%). Within the lesbian/gay group, those who had alcohol use dependence had a higher probability of having STDs $(8.73 \%)$ than those without alcohol use dependence (4.91\%). For bisexual individuals, those who had alcohol use dependence had a higher probability of having STDs (5.35\%) than those without alcohol use dependence $(3.56 \%)$. Heterosexual individuals who had alcohol use dependence had a higher probability of having STDs (3.30\%) than heterosexual individuals without alcohol use dependence $(1.83 \%)$.

Figure 3 shows that lesbian/gay individuals who had illicit drug use other than marijuana use dependence had the highest probability of having STDs (17.43\%) compared to bisexual 
Table 2 Adjusted odds ratios of self-reported STDs diagnosis status associated with sociodemographic characteristics, substance use dependence status, and MDE symptoms

\begin{tabular}{|c|c|c|}
\hline & AOR & $95 \% \mathrm{CI}$ \\
\hline \multicolumn{3}{|l|}{ Covariates } \\
\hline \multicolumn{3}{|l|}{ Age } \\
\hline $18-25$ & Ref & - \\
\hline $26-34$ & 0.90 & $(0.78,1.04)$ \\
\hline $35-49$ & 0.73 & $(0.63,0.84)$ \\
\hline $50-64$ & 0.66 & $(0.55,0.79)$ \\
\hline 65 or Older & 0.44 & $(0.34,0.56)$ \\
\hline \multicolumn{3}{|l|}{ Sex } \\
\hline Female & Ref & - \\
\hline Male & 0.73 & $(0.65,0.82)$ \\
\hline \multicolumn{3}{|l|}{ Race/ethnicity } \\
\hline Non-Hispanic White & Ref & - \\
\hline Non-Hispanic Black/African American & 1.29 & $(1.09,1.54)$ \\
\hline Hispanic & 0.79 & $(0.68,0.93)$ \\
\hline Other race & 0.82 & $(0.68,0.98)$ \\
\hline \multicolumn{3}{|l|}{ Sexual identity } \\
\hline Heterosexual (i.e., straight) & Ref & - \\
\hline Lesbian or Gay & 2.81 & $(2.24,3.54)$ \\
\hline Bisexual & 1.95 & $(1.60,2.37)$ \\
\hline \multicolumn{3}{|l|}{ Level of education completed } \\
\hline College graduate or higher & Ref & - \\
\hline Twelfth grade or less grade & 0.82 & $(0.67,0.99)$ \\
\hline High School diploma/GED & 0.94 & $(0.83,1.06)$ \\
\hline Some college credit but no degree & 1.02 & $(0.85,1.22)$ \\
\hline Associate's degree & 1.00 & $(0.82,1.23)$ \\
\hline \multicolumn{3}{|l|}{ Total family income } \\
\hline Less than $\$ 20,000$ & Ref & - \\
\hline$\$ 20,000-\$ 49,999$ & 0.91 & $(0.77,1.07)$ \\
\hline$\$ 50,000-\$ 74,999$ & 0.84 & $(0.70,1.02)$ \\
\hline$\$ 75,000$ or More & 0.77 & $(0.67,0.89)$ \\
\hline \multicolumn{3}{|l|}{ Employment status } \\
\hline Employed full time & Ref & \\
\hline Employed part time & 1.04 & $(0.88,1.23)$ \\
\hline Unemployed & 1.20 & $(0.93,1.54)$ \\
\hline Other & 0.79 & $(0.66,0.95)$ \\
\hline \multicolumn{3}{|l|}{ Independent variables } \\
\hline \multicolumn{3}{|l|}{ Past year MDE symptoms } \\
\hline No & Ref & \\
\hline Yes & 1.70 & $(1.46,1.99)$ \\
\hline \multicolumn{3}{|l|}{ Past year alcohol use dependence } \\
\hline No & Ref & - \\
\hline Yes & 1.79 & $(1.49,2.16)$ \\
\hline \multicolumn{3}{|l|}{$\begin{array}{l}\text { Past year illicit drug use other than mari- } \\
\text { juana use dependence }\end{array}$} \\
\hline No & Ref & \\
\hline Yes & 2.25 & $(1.73,2.92)$ \\
\hline \multicolumn{3}{|l|}{ Past year marijuana use dependence } \\
\hline No & Ref & - \\
\hline
\end{tabular}

Table 2 (continued)

\begin{tabular}{lll}
\hline & AOR & $95 \%$ CI \\
\hline Yes & $\mathbf{1 . 9 0}$ & $\mathbf{( 1 . 5 7 , 2 . 3 1 )}$ \\
\hline
\end{tabular}

Weighted $\mathrm{N}=247,549,178$ and unweighted $\mathrm{N}=127,584$

AOR, Adjusted odds ratio; $95 \% C I, 95 \%$ confidence interval; Statistically significant values at $p<0.05$ are in bold

Ref., Reference group

$M D E$, Major depressive episode

$S T D$, Sexually transmitted diseases

(6.44\%) and heterosexual (3.88\%) individuals with an illicit drug use other than marijuana use dependence. Among lesbians/gays, those who had illicit drug use other than marijuana use dependence had a higher probability of having STDs (17.43\%) than those without illicit drug use other than marijuana use dependence $(4.75 \%)$. For bisexuals, those who had illicit drug use other than marijuana use dependence had a higher probability of having STDs $(6.44 \%)$ than those without illicit drug use other than marijuana use dependence (3.57\%). Among heterosexuals, the probability of having STDs was higher for those with illicit drug use other than marijuana use dependence (3.88\%) than for those without illicit drug use other than marijuana use dependence (1.85\%). Across all sexual identity groups, the probability of having STDs was higher for those with illicit drug use other than marijuana use dependence (3.88\%-17.43\%) than for those with no illicit drug use other than marijuana use dependence $(1.85 \%-4.75 \%)$.

As shown in Fig. 4, the probability of having STDs was highest for bisexual individuals who had marijuana use dependence $(6.84 \%)$ than for lesbian/gay $(5.30 \%)$ and heterosexual (3.60\%) individuals with marijuana use dependence. Among the bisexual individuals, those who had marijuana use dependence had a higher probability of having STDs $(6.84 \%)$ than those without marijuana use dependence (3.52\%). For the lesbian/gay individuals, those who had marijuana use dependence had a higher probability of having STDs (5.30\%) than those without marijuana use dependence $(5.15 \%)$. Among the heterosexual individuals, the probability of having STDs was higher for those who had marijuana use dependence $(3.60 \%)$ than for those who had no marijuana use dependence (1.86\%). In general, individuals who had marijuana use dependence had the highest probability of having STDs (3.60-6.84\%\%) compared to those who had no marijuana use dependence (1.86-5.15\%).

\section{Discussion}

In the current study, we examined the association between STD diagnosis and MDE symptoms, and substance use. The study also examined differences in STD diagnosis 
Fig. 1 Differences in STDs diagnosis status between and within MDE symptoms and sexual identity

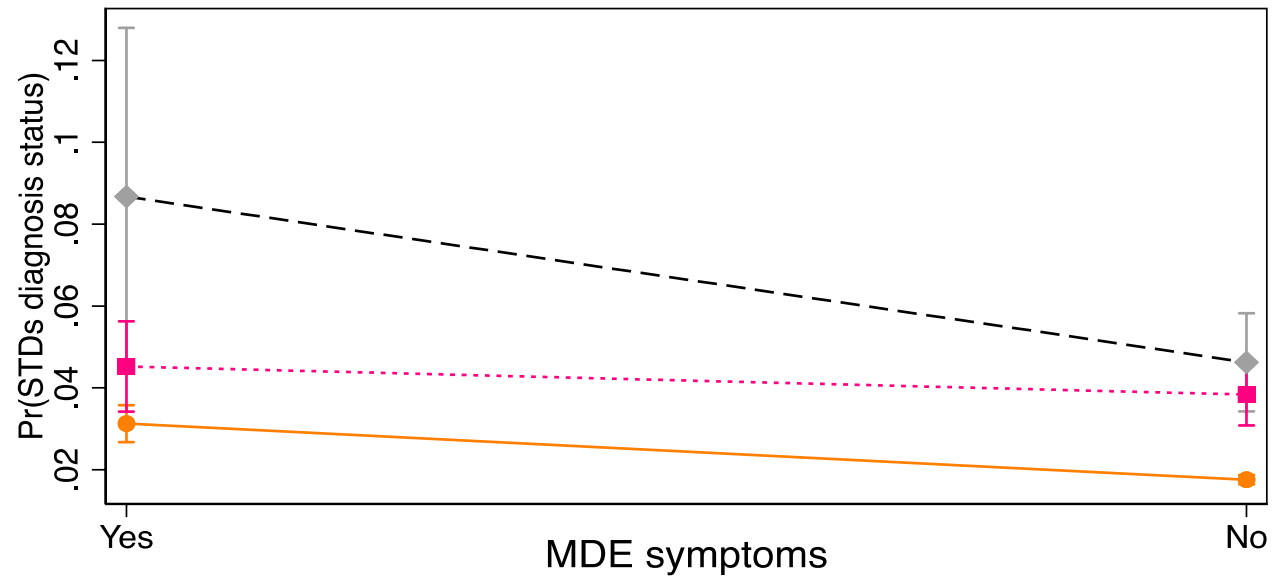

Sexual identity:

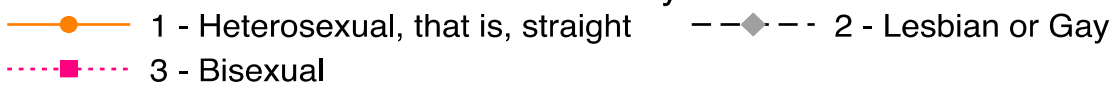

Fig. 2 Differences in STDs diagnosis status between and within alcohol use dependence status and sexual identity

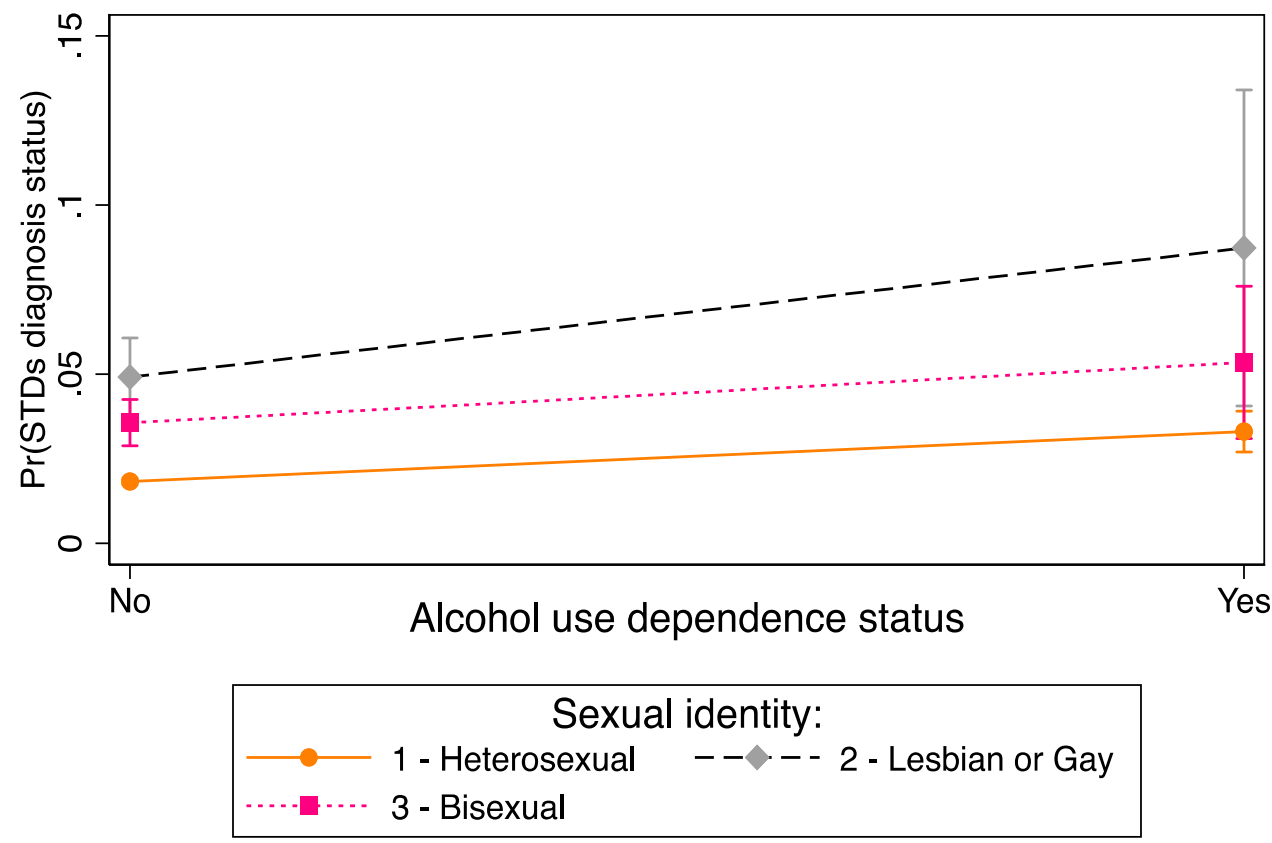

status between and within sexual identity groups (i.e., lesbian/gay, bisexual, and heterosexual persons) by MDE symptoms, alcohol use dependence, illicit drug use other than marijuana use dependence, and marijuana use dependence. The descriptive findings show that approximately $2 \%$ of the study population have STDs, where approximately $17 \%$ had MDE symptoms and less than onetenth of the population were dependent on substance use or were lesbian/gay. These rates suggest that people with mental health disorder symptoms, substance use dependence, and sexual minorities are likely to be burdened with STDs. This has several implications for public health interventions and policy directions to modify population lifestyles.

Our findings show that having MDE symptoms is associated with a higher likelihood of having an STD, suggesting that addressing depressive symptoms may potentially reduce the risk of STD contraction. Previous studies conducted among deploying shipboard US military personnel (Harbertson et al. 2019), individuals aged 18-28 years (Jenkins and Botchway 2016), and psychiatric outpatient sample (Carey et al. 2004), have shown an association between MDE symptoms and higher odds of having STD (Harbertson et al. 2019; Jenkins and Botchway 2016; Chen et al. 
Fig. 3 Differences in STDs diagnosis status between and within illicit drug use other than marijuana use dependence status and sexual identity

Fig. 4 Differences in STDs diagnosis status between and within marijuana use dependence status and sexual identity

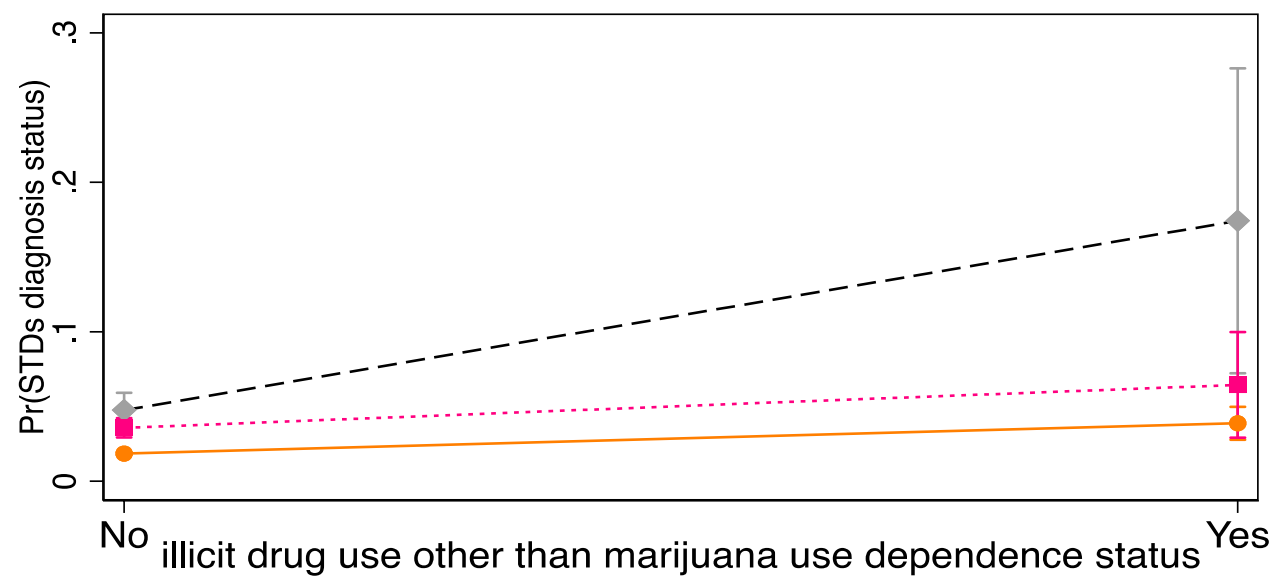

Sexual identity:

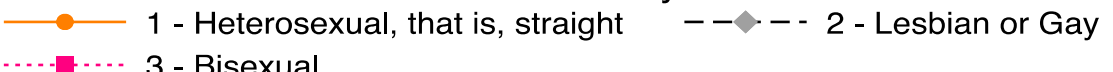

...-… 3 - Bisexual

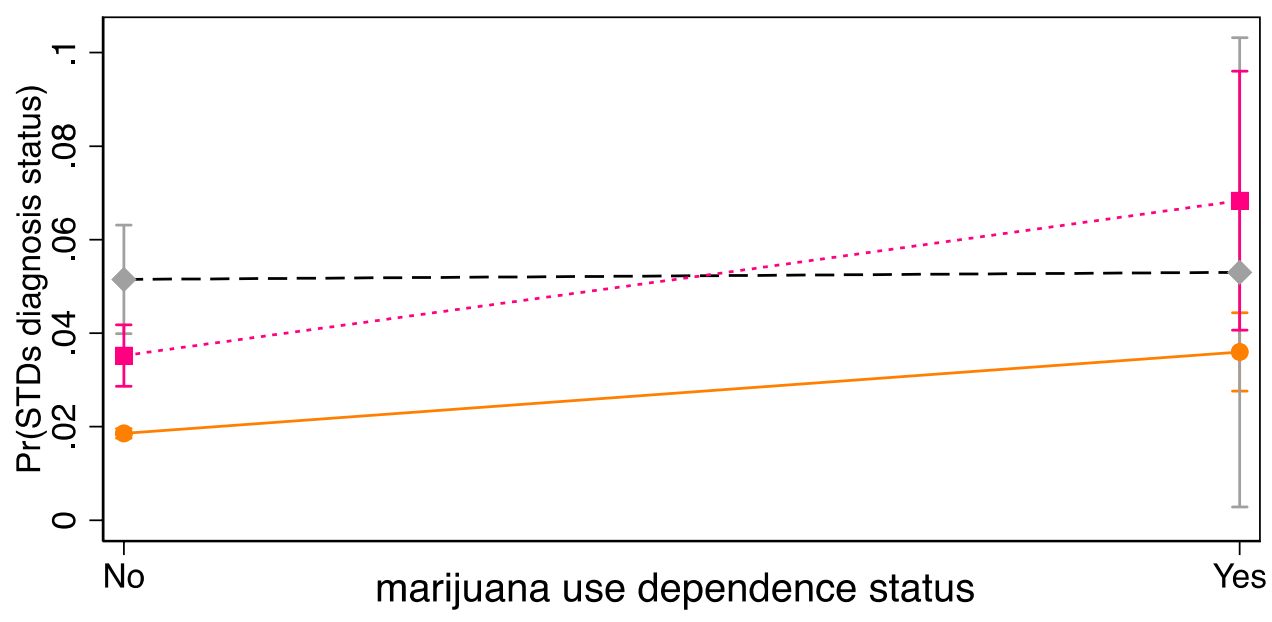

Sexual identity:

1 - Heterosexual, that is, straight $\quad----2$ - Lesbian or Gay
2008; Carey et al. 2004). This may imply that depressive symptoms may play a significant part in STD contractions irrespective of population characteristics such as age, being military or civilian, and other psychiatric behaviors. Our findings further show that individuals who are dependent on alcohol use or illicit drug use are more likely to be infected with STDs. This is consistent with other studies that linked substance use with disproportionately higher STD diagnoses among various populations (Feaster et al. 2016; Khan et al. 2013; Cook and Clark 2005), but these studies included only patients at clinics and young adults. This notable disadvantage in STD diagnosis has been linked to the fact that individuals with MDE symptoms and alcohol dependence as well as illicit drug use are prone to risky sexual behaviors such as multiple sexual partnerships and unprotected sexual intercourse, which expose them to higher STD risks (Coyle et al. 2019; Jenkinson et al. 2014; Khan et al. 2013). Consistent with previous studies (CDC 2021; Kreisel et al. 2021; CDC 2019; Hogben and Leichliter 2008), our findings suggest that females, people aged 18-25 years, nonHispanic Black/African Americans, and people who have less than $\$ 20,000$ annual total family income are at higher risks of having STD. This implies that underserved populations such as racial/ethnic minorities, low-income populations, and young people have high burdens of STDs, suggesting the need to treat those with STDs and strengthen 
sexual educational campaigns to reduce and prevent STDs among these populations. These findings may explain why young people, including those aged 18-24 years, have been found to contribute almost half of the new STD infections reported in 2018 (CDC 2021). While men and women are significantly affected by STDs, estimates showed women compared to men had more prevalent cases across data collected for eight STDs in 2018 (Kreisel et al. 2021). This may be because women have heightened susceptibility to infection due to broader cervical ectopy (Healthy People 2021). Women also suffer more STD complications with at least 24,000 US women becoming infertile due to undiagnosed or untreated STDs (Healthy People 2021).

In another analysis of examining whether the association between STDs diagnosis status and sexual identity is influenced by mental health and substance use disorder symptoms, we found significant differences in STD diagnosis between and within sexual identity subgroups due to MDE symptoms, alcohol, illicit drug use other than marijuana, and marijuana use dependence. Our findings suggest that the risk of STD is significantly higher among lesbian/gay (but not bisexual) individuals who have MDE symptoms. Although an elevated STD risk exists among sexual and gender minority (SGM) individuals irrespective of their MDE symptom status, those with MDE symptoms have higher risks of contracting STDs. Similarly, STD risks are higher among people who are dependent on alcohol, illicit drugs, or marijuana use, but the STD risk levels are significantly greater among lesbian/gay and bisexual individuals who are dependent on alcohol, illicit drug, or marijuana use than heterosexual individuals. Studies have indicated that men who have sex with men and women who have sex with women have greater substance use rates than heterosexual individuals (McCabe et al. 2009; Cochran et al. 2004). In addition, similar to MDE symptoms, substance abuse has been strongly linked to various risky sexual behaviors, which leads to a higher risk of STD diagnosis (Santos et al. 2018; Sutarso et al. 2016; Khan et al. 2013; Hutton et al. 2008). A possible explanation for these differences is that sexual and gender minority individuals have higher health-related problems, including STDs, MDE symptoms, and substance or drug use dependence, due to healthcare disparities associated with prejudice, unfair treatment, and discrimination (Jennings et al. 2019; Albuquerque et al. 2016; Logie 2012; Heck et al. 2006). Various previous studies have also associated same-sex and bisexual individuals with greater STD risks than heterosexual individuals (Ritter and Ueno 2019; Tao 2008), although only US women aged 15 to 44 years and older adults were examined in these studies. The intersectionality of substance abuse, in all forms, and sexual and gender minority identity may play a vital role in STD levels in the general population. Our findings, therefore, provide knowledge on the significant implications of the interplay of MDE symptoms or substance use dependence and sexual minority identity for STD diagnosis in the general population that can be incorporated in STD prevention programs and policies, particularly among sexual minorities.

The current study has a few potential limitations that may impinge on the findings. Given the cross-sectional nature of the data, we were unable to assess a temporal sequence or causal relationships to determine whether STD acquisition precedes substance use and mental health disorders. Therefore, we reported associations and did not make causal inferences in this study. Also, this study did not examine the various types or categories of STD, which may have different risk levels. Furthermore, the NSDUH was self-reported data and, hence, is prone to response and recall biases from participants which may result in over or underreporting and may affect our findings. Additionally, the NSDUH publicuse data does not include a variable for lesbian women and gay men to compare differences in STD prevalence between them. Hence, we were unable to assess this comparison in our study. Notwithstanding, the NSDUH questions used to examine the self-reported responses have been widely adopted and applied. In addition, the study drew on large nationally representative data to enhance the accuracy of estimations and generalizability of our findings to the United States population. Consequently, the study provides a vital contribution to knowledge by showing differences in STD diagnosis across sexual identity subgroups and their MDE and substance use dependence status.

\section{Conclusions}

In this study, we provide evidence that young people, nonHispanic Blacks, low-income populations, MDE symptoms, and dependence on alcohol and illicit drug use are significantly associated with increased STD diagnosis in the general population. As well, sexual and gender minority (SGM) individuals are excessively disadvantaged in STD risk levels compared with heterosexual adults. Examining the between and within-group inequalities in STD diagnosis helps to better understand and identify the higher risk population subgroups for the direction of public health policies and resources to address the risk levels. The study suggests the need to develop tailored interventions and programs to reduce MDE symptoms and substance use dependence which will consequently reduce the prevalence of STD, especially among SGM individuals. Future studies may use prospective data to examine temporal effects to better understand whether STD infection precedes substance use and mental health disorders while also studying the differences in STD prevalence between lesbian and gay individuals. 
Author contributions David Adzrago served as the leading author, conceived the study, performed the statistical analyses, conducted the literature review, drafted the manuscript, and coordinated writing the manuscript. Samuel H. Nyarko and Nnenna Ananaba conducted the literature review and contributed to the writing of the manuscript. Christine Markham provided critical revisions to the manuscript. All authors reviewed the manuscript and agreed to the publication of the manuscript.

Availability of data and material Publicly available data and material at https://www.samhsa.gov/data/data-we-collect/nsduh-natio nal-survey-drug-use-and-health.

Code availability Not applicable.

\section{Declarations}

Conflicts of interest All authors have no conflicts of interest to disclose that are relevant to the content of this article.

Ethics approval This paper was performed using de-identified data and therefore a review from the authors' Institutional Review Board was not required.

\section{Consent to participate Not applicable.}

Consent for publication Not applicable.

Open Access This article is licensed under a Creative Commons Attribution 4.0 International License, which permits use, sharing, adaptation, distribution and reproduction in any medium or format, as long as you give appropriate credit to the original author(s) and the source, provide a link to the Creative Commons licence, and indicate if changes were made. The images or other third party material in this article are included in the article's Creative Commons licence, unless indicated otherwise in a credit line to the material. If material is not included in the article's Creative Commons licence and your intended use is not permitted by statutory regulation or exceeds the permitted use, you will need to obtain permission directly from the copyright holder. To view a copy of this licence, visit http://creativecommons.org/licenses/by/4.0/.

\section{References}

Albuquerque GA, de Lima GC, da Silva QG, Alves MJ, Belém JM, dos Santos Figueiredo FW, da Silva PL, do Nascimento VB, da Silva Maciel E, Valenti VE, de Abreu LC (2016) Access to health services by lesbian, gay, bisexual, and transgender persons: systematic literature review. BMC Int Health Hum Rights 16(1):1-0

American Psychiatric Association (1994) Diagnostic and statistical manual of mental disorders (DSM-IV). American Psychiatric Association, Washington, DC

American Psychiatric Association (2013) Diagnostic and statistical manual of mental disorders (DSM-5®). American Psychiatric Association, Washington, DC

Carey KB, Senn TE, Walsh JL, Scott-Sheldon LA, Carey MP (2016) Alcohol use predicts number of sexual partners for female but not male STI clinic patients. AIDS Behav 20(1):52-59

Carey MP, Carey KB, Maisto SA, Schroder KE, Vanable PA, Gordon CM (2004) HIV risk behavior among psychiatric outpatients: association with psychiatric disorder, substance use disorder, and gender. J Nerv Ment Dis 192(4):289
CDC (2019) STDs in racial and ethnic minorities. 2018. Available at https://www.cdc.gov/std/stats18/minorities.htm. Accessed 26 Apr 2021

CDC (2021) STI Prevalence, Incidence, and Cost Estimates. Available at https://www.cdc.gov/std/statistics/prevalence-incidence-cost2020.htm. Accessed 5 May 2021

Center for Behavioral Health Statistics and Quality (2020a) 2019 National Survey on Drug Use and Health: Methodological summary and definitions. Available at https://www.samhsa.gov/data/. Accessed 20 Feb 2021

Center for Behavioral Health Statistics and Quality (2020b) 2019 National Survey on Drug Use and Health Public Use File Codebook. Available at https://www.samhsa.gov/data/. Accessed 20 Feb 2021. https://www.datafiles.samhsa.gov/sites/default/files/ field-uploads-protected/studies/NSDUH-2019/NSDUH-2019datasets/NSDUH-2019-DS0001/NSDUH-2019-DS0001-info/ NSDUH-2019-DS0001-info-codebook.pdf

Chen Y, Wu J, Yi Q, Huang G, Wong T (2008) Depression associated with sexually transmitted infection in Canada. Sex Transm Infect 84(7):535-540

Cochran SD, Ackerman D, Mays VM, Ross MW (2004) Prevalence of non-medical drug use and dependence among homosexually active men and women in the US population. Addiction 99(8):989-998

Cook RL, Clark DB (2005) Is there an association between alcohol consumption and sexually transmitted diseases? A systematic review. Sexually transmitted diseases 32(3):156-164

Coyle RM, Lampe FC, Miltz AR, Sewell J, Anderson J, Apea V, Collins S, Dhairyawan R, Johnson AM, Lascar M, Mann S (2019) Associations of depression and anxiety symptoms with sexual behaviour in women and heterosexual men attending sexual health clinics: a cross-sectional study. Sex Transm Infect 95(4):254-261

Dembo R, Belenko S, Childs K, Wareham J (2009) Drug use and sexually transmitted diseases among female and male arrested youths. J Behav Med 32(2):129-141

Erbelding EJ, Hummel B, Hogan T, Zenilman J (2001) High rates of depressive symptoms in STD clinic patients. Sex Transm Dis 28(5):281-284

Feaster DJ, Parish CL, Gooden L, Matheson T, Castellon PC, Duan R, Pan Y, Haynes LF, Schackman BR, Malotte CK, Mandler RN (2016) Substance use and STI acquisition: secondary analysis from the AWARE study. Drug Alcohol Depend 169:171-179

Harbertson J, Scott PT, Lemus H, Michael NL, Hale BR (2019) Cross-sectional study of sexual behavior, alcohol use, and mental health conditions associated with sexually transmitted infections among deploying shipboard US military personnel. Mil Med 184(11-12):e693-e700

Harvard School of Medicine (2005) National Comorbidity Survey (NCS). Available at https://www.hcp.med.harvard.edu/ncs/. Accessed 21 Jan 2021

Healthy People (2021) Sexually Transmitted Diseases I Healthy People 2020. Available at https://www.healthypeople.gov/2020/topicsobjectives/topic/sexually-transmitted-diseases. Accessed 9 Apr 2021

Heck JE, Sell RL, Gorin SS (2006) Health care access among individuals involved in same-sex relationships. Am J Public Health 96(6):1111-1118

Hogben M, Leichliter JS (2008) Social determinants and sexually transmitted disease disparities. Sex Transm Dis 35(12):S13-S18

Hutton HE, McCaul ME, Santora PB, Erbelding EJ (2008) The relationship between recent alcohol use and sexual behaviors: gender differences among sexually transmitted disease clinic patients. Alcohol Clin Exp Res 32(11):2008-2015

Jenkins WD, Botchway A (2016) Young adults with depression are at increased risk of sexually transmitted disease. Prev Med 88:86-89 
Jenkinson R, Bowring A, Dietze P, Hellard M, Lim MS (2014) Young risk takers: alcohol, illicit drugs, and sexual practices among a sample of music festival attendees. J Sex Transm Dis 2014:1-6

Jennings L, Barcelos C, McWilliams C, Malecki K (2019) Inequalities in lesbian, gay, bisexual, and transgender (LGBT) health and health care access and utilization in Wisconsin. Prev Med Rep 14:100864

Kaiser Family Foundation (2021) Substance use and risky sexual activity. Available at http://www.kff.org. Accessed 5 Apr 2021

Khan MR, Berger A, Hemberg J, O'Neill A, Dyer TP, Smyrk K (2013) Non-injection and injection drug use and STI/HIV risk in the United States: the degree to which sexual risk behaviors versus sex with an STI-infected partner account for infection transmission among drug users. AIDS Behav 17(3):1185-1194

Kreisel KM, Spicknall IH, Gargano JW, Lewis FM, Lewis RM, Markowitz LE, Roberts H, Johnson AS, Song R, Cyr SB, Weston EJ (2021) Sexually transmitted infections among US women and men: prevalence and incidence estimates, 2018. Sex Transm Dis 48(4):208-214

Logie C (2012) The case for the World Health Organization's commission on the social determinants of health to address sexual orientation. Am J Public Health 102(7):1243-1246

Magidson JF, Blashill AJ, Wall MM, Balan IC, Wang S, Lejuez CW, Blanco C (2014) Relationship between psychiatric disorders and sexually transmitted diseases in a nationally representative sample. J Psychosom Res 76(4):322-328

McCabe SE, Hughes TL, Bostwick WB, West BT, Boyd CJ (2009) Sexual orientation, substance use behaviors and substance dependence in the United States. Addiction 104(8):1333-1345

Newman LM, Berman SM (2008) Epidemiology of STD disparities in African American communities. Sex Transm Dis 35(12):S4-12

Otto-Salaj LL, Stevenson LY (2001) Influence of psychiatric diagnoses and symptoms on HIV risk behavior in adults with serious mental illness. AIDS Read 11(4):197-204

Ramrakha S, Caspi A, Dickson N, Moffitt TE, Paul C (2000) Psychiatric disorders and risky sexual behaviour in young adulthood: cross sectional study in birth cohort. Bmj 321(7256):263-266

Rein DB, Anderson LA, Irwin KL (2004) Mental health disorders and sexually transmitted diseases in a privately insured population. Am J Manag Care 10(12):917-924

Reisner SL, Mimiaga M, Case P, Grasso C, O'Brien CT, Harigopal P, Skeer M, Mayer KH (2010) Sexually transmitted disease (STD) diagnoses and mental health disparities among women who have sex with women screened at an urban community health center, Boston, MA, 2007. Sex Transm Dis 37(1):5

Ritter LJ, Ueno K (2019) Same-sex contact and lifetime sexually transmitted disease diagnoses among older adults. J Aging Health 31(6): 1043-1064

Rohde P, Noell J, Ochs L, Seeley JR (2001) Depression, suicidal ideation and STD-related risk in homeless older adolescents. J Adolesc 24(4):447-460

Santos GM, Rowe C, Hern J, Walker JE, Ali A, Ornelaz M, Prescott M, Coffin P, McFarland W, Raymond HF (2018) Prevalence and correlates of hazardous alcohol consumption and binge drinking among men who have sex with men (MSM) in San Francisco. PLoS One 13(8):e0202170

Shannon CL, Klausner JD (2018) The growing epidemic of sexually transmitted infections in adolescents: a neglected population. Curr Opin Pediatr 30(1):137-143

StataCorp LL (2019) Stata statistical software. Release 16.[software]. College Station, TX

Substance Abuse and Mental Health Services Administration (2020) Results from the 2019 National Survey on drug use and health: detailed tables. Center for Behavioral Health Statistics and Quality, Substance Abuse and Mental Health Services Administration, Rockville, MD. Available at https://www.samhsa.gov/data/report/ 2019-nsduh-detailed-tables. Accessed 20 Feb 2021

Sutarso T, Tang TL, Anerin DR, McCartt IW, Gibson CB (2016) Sexual temptation: substance abuse, no sex, safe sex, risky sex, and STDs. Int J Adolesc Med Health 30(5)

Tao G (2008) Sexual orientation and related viral sexually transmitted disease rates among US women aged 15 to 44 years. Am J Public Health 98(6):1007-1009

World Health Organization (2021) Sexually transmitted infections (STIs). Available at https://www.who.int/news-room/fact-sheets/ detail/sexually-transmitted-infections-(stis). Accessed 5 Apr 2021

Publisher's note Springer Nature remains neutral with regard to jurisdictional claims in published maps and institutional affiliations. 